\title{
ORTODOXIA Y FISURAS EN EL DISEÑO Y EJECUCIÓN DE ESTUDIOS DESCRIPTIVOS
}

\author{
JAIBERTH ANTONIO CARDONA-ARIAS \\ ${ }^{1}$ Microbiólogo y Bioanalista, MSc.,Epidemiología. \\ Grupo de Investigación Salud y Sostenibilidad, Escuela de Microbiología. \\ Universidad de Antioquia Medellín, Colombia. Facultad de Medicina Universidad Cooperativa de Colombia \\ Correspondencia: jaiberthcardona@gmail.com
}

Recibido: 6 Mayo 2014 Aceptado: 21 Diciembre 2014

\begin{abstract}
Resumen
Introducción: En diversos contextos se alude la investigación descriptiva en términos peyorativos por endilgarle sesgos e impacto exiguo en el estudio de factores de riesgo. Esto podría atribuirse al desconocimiento de sus fundamentos conceptuales y metodológicos.

Objetivo: Recabar las ventajas de los estudios descriptivos a partir de una revisión de sus fundamentos conceptuales y metodológicos.

Métodos: Revisión narrativa sobre las ventajas, usos y limitaciones de los estudios descriptivos, sumado al análisis de algunos aspectos metodológicos.

Resultados: Se presentan las particularidades de los estudios descriptivos en términos de sus tipologías, usos, ventajas y limitaciones. En relación con la fase conceptual de toda investigación se analizan las características específicas del problema, justificación, objetivos y marco teórico de un estudio descriptivo. En la fase metodológica se contrastan algunos autores que han estudiado temas determinantes para garantizar la validez de la investigación descriptiva como la clasificación y control de sesgos, la identificación de la confusión y el análisis estadístico. Al finalizar, se hace explícito el contenido de un artículo descriptivo a la luz de sus exigencias epidemiológicas y estadísticas.

Conclusión: La investigación descriptiva es la que expone de manera más veraz y directa la realidad de un fenómeno de estudio, por ello es necesario conocer sus exigencias conceptuales y operativas para mitigar concepciones erradas sobre su alcance.

Palabras clave: Epidemiología descriptiva; Metodología; Investigación operativa; Revisión; Diseño de Investigaciones Epidemiológicas.
\end{abstract}

\section{ORTHODOXY AND FISSURES IN THE DESIGN AND IMPLEMENTATION OF DESCRIPTIVE STUDIES}

\begin{abstract}
Introduction: In some contexts the descriptive researching has been referred with derogatory terms by its biases and low impact in the study of risk factors. This could be attributed to a lack of conceptual and methodological foundations.

Objective: To seek the advantages of the descriptive studies from a review of its conceptual and methodological foundations.
\end{abstract}


Methods: Narrative review of the benefits, uses and limitations of descriptive studies, coupled with the analysis of some methodological aspects.

Results: the specific items of descriptive studies are presented in terms of their types, uses, advantages and limitations. Regarding the conceptual phase of the research was analyzed the problem, grounds, objectives and theoretical framework. In the methodological phase are presented some determinants to ensure validity of descriptive researching as the classification and control of bias, confounding and statistical analysis. At the end it is showed the content of a descriptive article in the light of their needs and epidemiological statistics.

Conclusion: Descriptive researching is exposing more accurate and directly the reality of a phenomenon under study, so it is necessary to understand its conceptual and operational requirements to mitigate misconceptions about its scope.

Keywords: Epidemiology, Descriptive; Methods; Operations Research; Review; Epidemiologic Research Design.

\section{ORTODOXIA E FISSURAS NO PROJETO E EXECUÇÃO DE ESTUDOS DESCRITIVOS}

\section{Resumo}

Introdução: Em muitos contextos se fala pejorativamente da investigação por descritiva impingirlhe sesgos e pouco impacto no estudo de fatores de resgo. Isso pode ser atribuído ao desconhecimento dos fundamentos conceptuais e metodológicos.

Objetivo: Coletar as vantagens dos estudos descritivos com uma revisão dos fundamentos conceptuais e metodológicos.

Métodos: Revisão narrativa das vantagens, fusos e limitações dos estudos descritivos, mais o análises de alguns aspetos metodológicos.

Resultados: Presentam-se as particularidades dos estudos descritivos em términos de suas tipologias, aplicações, vantagens e limitações. Em relação com a fase conceptual de toda investigação, as características específicas do problema, justificação, objetivos e marco teórico dum estudo descritivo foram analisados. Na fase metodológica alguns autores que tem tratado o tema foram contrastados. Eles tem estudado temas determinantes para garantir a validez da investigação descritiva como a classificação y controle de riscos, a identificação da confusão $e$ o análises estadístico. Ao final se fiz claro o conteúdo duma apresentação descritiva guiado pelas exigências epidemiológicas e estadísticas.

Conclusão: A investigação descritiva é a que mostra da melhor maneira a realidade dum fenómeno de estudo. Assim é preciso conhecer seus exigências conceptuais e operativas para mitigar conceitos errados no alcance.

Palavras-chave: epidemiologia descritiva, metodologia, investigação operativa; Projetos de Pesquisa Epidemiológica y Revisão. 


\section{Introducción}

Algunas tendencias investigativas actuales abogan por estudios preclínicos (o de investigación básica), analíticos y experimentales que, al conformar los grupos de estudio con base en criterios de inclusión y exclusión exigentes, no dan cuenta de las formas en que "naturalmente" se distribuye el fenómeno de estudio; esto podría evidenciarse en el hecho que algunos programas académicos de posgrado, entes financiadores de investigación, revistas científicas o comunidades académicas, han circunscrito la investigación descriptiva al pregado, a semilleros de investigación o a investigadores noveles; con la subsecuente subvaloración de su relevancia. Con este tipo de posturas se obstan aspectos relevantes como el que la investigación descriptiva es la más próxima a la realidad del fenómeno estudiado, dado que su finalidad es determinar qué está ocurriendo y cuáles son los subgrupos más afectados; investigando múltiples factores de exposición e incluso múltiples efectos (1).

Lo anterior puede suponer tres tipos de obstáculos que presenta el desarrollo de la investigación descriptiva: i) bajo conocimiento de su fundamentación teórica, ii) limitaciones en el cumplimiento de sus exigencias metodológicas y iii) necesidad de mejorar el uso de algunas herramientas estadísticas.

Las falencias teórico-conceptuales que se presentan en diversos contextos científicos y académicos sobre la investigación descriptiva, pueden reflejarse en el hecho que algunos le endilgan múltiples sesgos, impacto exiguo en el estudio de la causalidad y generación de recomendaciones científicas de baja calidad; lo que ha derivado en calificativos peyorativos que no se corresponden con su relevancia en la orientación de acciones preventivas y de intervención.

Por otra parte, para quienes realizan estudios descriptivos, existen exigencias adicionales al manejo conceptual, que están relacionadas con el rigor epidemiológico, estadístico y de otros temas relacionados con programas de metodología de la investigación. En el tercer obstáculo, existe la necesidad de evaluar algunos retos en las mediciones y análisis estadísticos que algunos académicos han circunscrito a la investigación analítica y experimental, como los modelos multivariables.

Por lo anterior, el objetivo de este artículo es recabar las ventajas de los estudios descriptivos a partir de la revisión de sus fundamentos conceptuales y metodológicos, sobre la base que éstos tradicionalmente no se encuentran agregadas en un único texto sino que se atomizan en textos de epidemiología, estadística o metodología de la investigación, dificultando la comprensión de sus bondades.

\section{Materiales y métodos}

Revisión narrativa de artículos y libros de metodología de la investigación, epidemiología y estadística para caracterizar los manuscritos con base en la siguiente estructura:

Tabla 1. Estructura para la caracterización de manuscritos

\begin{tabular}{|l|l|l|}
\hline \multirow{2}{*}{$\begin{array}{l}\text { Fundamentación } \\
\text { conceptual }\end{array}$} & \multicolumn{2}{|l|}{ Definición y Tipología } \\
\cline { 2 - 3 } & Usos, Ventajas y limitaciones \\
\hline \multirow{2}{*}{$\begin{array}{l}\text { Consideraciones } \\
\text { metodológicas }\end{array}$} & $\begin{array}{l}\text { Fase } \\
\text { metodológica }\end{array}$ & $\begin{array}{l}\text { Problema, Justificación, Objetivos y } \\
\text { Marco teórico }\end{array}$ \\
\cline { 2 - 3 } & $\begin{array}{l}\text { Tamaño de muestra, Recolección de } \\
\text { la información, Control de sesgos, } \\
\text { Cuantificación de la confusión y } \\
\text { Análisis estadístico }\end{array}$ \\
\cline { 2 - 3 } & $\begin{array}{l}\text { Fase de } \\
\text { divulgación }\end{array}$ & $\begin{array}{l}\text { Contenido del artículo y Guías para el } \\
\text { reporte de un estudio transversal }\end{array}$ \\
\hline
\end{tabular}

A partir de la tabla anterior se realizó la presentación de resultados.

\section{Resultados}

Fundamentación conceptual: tipología, ventajas y limitaciones.

Por definición, la investigación descriptiva estudia un fenómeno en condiciones naturales sin considerar hipótesis, es decir, este diseño no permite corroborar o falsear hipótesis causales (solo descriptivas o de exploración de asociaciones) sino que las genera como base para los estudios analíticos (2-3).

La mayoría de los estudios descriptivos se clasifican como transversales dado que la medición del evento bajo estudio (variable de respuesta, desenlace o dependiente) es simultánea con la medición de las variables independientes o de exposición, a diferencia de los longitudinales donde la medición de la exposición y el evento se dan en momentos diferentes. Se dividen en estudios de prevalencia, de incidencia, estudios de caso o series de casos cuando la unidad de análisis es cada individuo (4); o en estudios ecológicos cuando la unidad de análisis es un grupo, comunidad o población geográficamente bien delimitadas (mediciones agregadas, ambientales o globales). Los estudios ecológicos presentan las siguientes variantes:

1. Exploratorios: investigan relaciones entre variables de acuerdo con el marco teórico, comparando el evento de estudio en un lugar en diferentes momentos, o comparando el evento en el mismo periodo en lugares diferentes (5).

2. Grupos múltiples: son próximos a un estudio 
analítico al comparar el evento con algún factor de exposición en diferentes grupos o poblaciones (5).

3. Series de tiempo: en dos o más poblaciones, estudian y comparan la exposición a variables ambientales, físicas, socioeconómicas o de salud, en diferentes periodos (5).

4. Estudios mixtos: son producto de una combinación de los anteriores (5).

Las mayores ventajas de los estudios descriptivos incluyen:

1. Generación de hipótesis.

2. Establece la magnitud del problema e identifica posibles factores de riesgo.

3. Estudia asociaciones entre múltiples exposiciones y múltiples eventos.

4. Útiles para estudiar eventos de larga duración y curso lento.

5. Bajo costo y rapidez.

6. Son la base para la planificación y administración de los servicios de salud y estimar las necesidades asistenciales (3).

\section{Limitaciones:}

1. Dado que las variables dependientes e independientes generalmente se miden en el mismo momento, no es posible establecer asociaciones causales (no es posible determinar si la exposición antecedió al evento), exceptuando fenómenos donde la exposición no cambia en el tiempo o donde la teoría indica la obviedad en la antecedencia del factor de riesgo.

2. No es plausible su aplicación en eventos de baja frecuencia o corta duración, porque pueden generar subestimaciones.

3. Presenta múltiples sesgos potenciales: de duración (cuando la exposición o el evento es de corta duración y por ello no se detecta su ocurrencia en la realización del estudio), de selección (los más o los menos afectados por el problema no participan del estudio), de cortesía (el sujeto de estudio da respuestas pensando en lo cree que el encuestador aprueba), de vigilancia (el evento se confirma mejor en la población de estudio que en la población general), de memoria (al preguntar por algunos factores de exposición pasados, quienes presentan el evento pueden recordarlos más fácilmente que los no afectados por el problema) y de información debidos al uso de instrumentos de medición no validados, a problemas de los investigadores (no estandarizados, no capacitados) o problemas en los sujetos de estudio (por no cumplir condiciones mínimas para la recolección de la información como estar bajo el influjo de alucinógenos, problemas cognitivos, entre otros (3).

\section{Consideraciones metodológicas}

Fase conceptual: problema, justificación, objetivos, marco teórico.

Son múltiples los textos que existen sobre metodología de la investigación, en estos se explicitan algunos aspectos transversales a todo tipo de estudios, lo que resulta útil para las personas que no conocen mucho de esta área, pero presenta la gran limitación de no hacer explicitas la particularidades inherentes a cada tipo de estudio. En coherencia con la pretensión de este texto, a continuación se indican aspectos genéricos de metodología de la investigación y su aplicación en los estudios descriptivos (6).

Tabla 2. Elementos transversales a la fase conceptual de un proyecto de investigación y específicos para estudios descriptivos.

\begin{tabular}{|c|c|}
\hline \multicolumn{2}{|c|}{$\begin{array}{l}\text { Título: Es el tema de investigación más su delimitación poblacional (en quienes } \\
\text { se desarrollará), geográfica o espacial (donde) y temporal (cuando). Para el objetivo } \\
\text { general del estudio, se toma el título y se antecede de un verbo afín a los estudios } \\
\text { descriptivos como describir, determinar, estimar o establecer }\end{array}$} \\
\hline \multicolumn{2}{|c|}{ Planteamiento del problema } \\
\hline letodología genérica & $\begin{array}{l}\text { Consideraciones específicas para } \\
\text { estudios descriptivos }\end{array}$ \\
\hline $\begin{array}{l}\text { Elegir el contexto de } \\
\text { desarrollo del tema }\end{array}$ & $\begin{array}{l}\text { Se debe evidenciar que en la población estudiada se } \\
\text { desconoce la magnitud y distribución del fenómeno } \\
\text { de estudio. }\end{array}$ \\
\hline $\begin{array}{l}\text { Evaluar la deficiencia } \\
\text { en el conocimiento } \\
\text { del tema }\end{array}$ & $\begin{array}{l}\text { Sustentar el desconocimiento sobre lo que está } \\
\text { ocurriendo, la magnitud del fenómeno estudiado y } \\
\text { su distribución según algunas características de base } \\
\text { de la población. }\end{array}$ \\
\hline $\begin{array}{l}\text { Definir consecuencias } \\
\text { de la investigación }\end{array}$ & $\begin{array}{l}\text { Focalizarse en la necesidad de establecer hipótesis } \\
\text { causales para posteriores estudios analíticos. } \\
\text { Evidenciar cómo el desconocimiento de lo que está } \\
\text { ocurriendo conducirá a la formulación de acciones } \\
\text { intervenciones equivocadas. }\end{array}$ \\
\hline $\begin{array}{l}\text { La idea debe ayudar a } \\
\text { resolver problemas, } \\
\text { aportar conocimientos, } \\
\text { ser novedosa y generar } \\
\text { interrogantes }\end{array}$ & $\begin{array}{l}\text { Debe evidenciarse por qué no es oportuno } \\
\text { extrapolar los resultados de estudios similare } \\
\text { realizados en otros escenarios, es decir, sustentar las } \\
\text { particularidades de la población, lugar y tiempo de } \\
\text { estudio, que demuestran o ayuden a evidenciar que } \\
\text { sus características permiten suponer una magnitudy } \\
\text { distribución diferentes que la descrita en estudios } \\
\text { previos. }\end{array}$ \\
\hline $\begin{array}{l}\text { Relacionar dos o más } \\
\text { conceptos o variables }\end{array}$ & $\begin{array}{l}\text { En estudios descriptivos, los dos conceptos o } \\
\text { variables son el evento del cual se desea establecer } \\
\text { su magnitud, y las características de base de la } \\
\text { población o factores asociados al evento reportados } \\
\text { en la literatura. Estos últimos serán la base para } \\
\text { estudiar la distribución del evento, es decir, definir si } \\
\text { el problema tiene una distribución uniforme en todo } \\
\text { la población o varía en los subgrupos conformados } \\
\text { por las variables independientes (en cuyo se } \\
\text { identificarían grupos de mayor riesgo). Es oportuno } \\
\text { aclarar que este punto es el que justifica el uso de } \\
\text { pruebas de asociación estadística para explorar } \\
\text { factores relacionados con el evento, los cuales darán } \\
\text { origen a las hipótesis. }\end{array}$ \\
\hline
\end{tabular}




\begin{tabular}{|c|c|}
\hline $\begin{array}{l}\text { Tener contenido } \\
\text { empírico }\end{array}$ & $\begin{array}{l}\text { Se debe exponer antecedentes de estudios previos } \\
\text { que ayuden a tener un valor (o rango) aproximado } \\
\text { sobre la frecuencia (magnitud) del evento y sus } \\
\text { principales factores asociados, en otros contextos } \\
\text { similares Algunos autores consideran que este punto } \\
\text { es más propio de la justificación del estudio. }\end{array}$ \\
\hline \multicolumn{2}{|l|}{ Justificación } \\
\hline \multicolumn{2}{|c|}{ Académica, Tecnológica, Social y Económica } \\
\hline $\begin{array}{l}\text { Teórica o } \\
\text { epistemológica }\end{array}$ & $\begin{array}{l}\text { Desarrolla nuevo conocimientos, aporta evidencia a } \\
\text { los ya existentes, brinda fundamento para la } \\
\text { realización de nuevas investigaciones. La base es la } \\
\text { generación de hipótesis que puedan falsearse o } \\
\text { corroborarse en estudios analíticos posteriores. }\end{array}$ \\
\hline $\begin{array}{l}\text { Implicaciones } \\
\text { prácticas }\end{array}$ & $\begin{array}{l}\text { Hacer explícitas las acciones de prevención de la } \\
\text { enfermedad o promoción de la salud que podrían } \\
\text { derivarse del conocimiento de la magnitud del evento } \\
\text { y sus grupos de mayor riesgo (subgrupos de la } \\
\text { población que presentaron una mayor ocurrencia } \\
\text { del evento). En este punto se determina quién o } \\
\text { quiénes se beneficiarán con los resultados. }\end{array}$ \\
\hline \multicolumn{2}{|l|}{ Objetivos } \\
\hline \multicolumn{2}{|c|}{ Medibles, factibles y viables, coherentes con el diseño del estudio. } \\
\hline \multicolumn{2}{|l|}{ Marco teórico } \\
\hline $\begin{array}{l}\text { Indica las diferencias y } \\
\text { similitudes con estudios } \\
\text { previos. }\end{array}$ & $\begin{array}{l}\text { Ausencia de hipótesis para el contexto en que se } \\
\text { desea realizar el estudio, ello evidenciaría la } \\
\text { necesidad de hacer la investigación descriptiva. }\end{array}$ \\
\hline \multicolumn{2}{|c|}{ Ubica la investigación en un campo determinado del conocimiento actual. } \\
\hline \multicolumn{2}{|c|}{ Conceptúa las variables del estudio y ayuda a identificarlas. } \\
\hline \multicolumn{2}{|c|}{$\begin{array}{l}\text { Describe cómo los resultados contribuirán al conocimiento o práctica en que se } \\
\text { inserta el proyecto: dudas y controversias que ayudará a esclarecer. }\end{array}$} \\
\hline \multicolumn{2}{|c|}{ Orienta el estudio y documenta la necesidad de realizarlo. } \\
\hline $\begin{array}{l}\text { Ayuda a prevenir er } \\
\text { investigación. }\end{array}$ & provee el sustento teórico del problema de \\
\hline
\end{tabular}

En el objetivo general debe hacerse explícito que la pretensión central es describir o determinar la ocurrencia del evento y su distribución según algunas características de la población de estudio; aunado a la delimitación poblacional, geográfica y temporal. Generalmente se trabaja con una población o grupo único, aunque se debe aclarar que algunos estudios descriptivos incluyen varios grupos, pero con el fin de establecer y comparar la magnitud (y distribución) del evento en varios grupos o momentos; a diferencia de los estudios analíticos en los cuales la conformación de los grupos se hace con base en una factor asociado para falsear o corroborar asociaciones causales.

Los estudios descriptivos plantean tres o cuatro tipos de objetivos específicos:

1. Describir las características de base de la población de estudio; aquellas que puedan influenciar la distribución del evento y que estén respaldadas por el marco teórico.

2. Determinar la magnitud del evento.
3. Explorar la asociación entre las características del grupo de estudio y el evento investigado.

En este tipo de estudio no se recomienda poner sólo "Asociar" o "Relacionar" o "Analizar" la magnitud del evento según las características de la población de estudio, ya que estos términos aluden asociaciones de tipo causal, las cuales solo se pueden lograr en estudios analíticos, en los que se puede demostrar que los factores de exposición anteceden la presentación del evento. Dado que este último punto no puede garantizarse en un estudio descriptivo, se emplea la expresión "explorar la asociación" para que sea explícito que las posibles asociaciones estadísticas halladas no son causales sino exploratorias; esto constituye la base para la formulación de hipótesis y demostrar que el evento no presenta una distribución uniforme en la población sino que existen grupos con una presentación mayor del evento.

4. Identificar las características de la población que podrían explicar la ocurrencia del evento, o determinar las características de la población que presentan mayor importancia en la ocurrencia del evento.

La necesidad de formular este último objetivo está dada en que los estudios descriptivos, por definición, se emplean para investigar múltiples exposiciones, de las cuales no todas presentan la misma importancia, por ello se debe hacer un modelo multivariado (regresiones) cuyos coeficientes indican la mayor o menor injerencia que tienen las variables independientes sobre la presentación del evento. Además, en el caso que existen varias variables independientes que presenten asociación estadística con el evento, se debe hacer un ajuste multivariable para descartar posibles variables de confusión.

Tabla 3. Ejemplo de la formulación de objetivos en un estudio descriptivo.

\section{Ejemplo 1}

Objetivo general: Determinar la prevalencia de ansiedad y su asociación con características demográficas y socioeconómicas en docentes de una universidad de Medellín, 2015.

\begin{tabular}{|l|l|}
\hline Objetivos específicos & \multicolumn{1}{|c|}{ Plan de análisis } \\
\hline $\begin{array}{l}\text { Describir las } \\
\text { características } \\
\text { demográficas y } \\
\text { socioeconómicas del } \\
\text { grupo de estudio. }\end{array}$ & $\begin{array}{l}\text { Estas características incluyen variables cualitativas y } \\
\text { cuantitativas, el plan análisis univariado diría: la } \\
\text { descripción se realizará por medio de frecuencias } \\
\text { absolutas (\#) y relativas (\%) para las variables } \\
\text { cualitativas y medidas de resumen para las } \\
\text { cuantitativas. }\end{array}$ \\
\hline $\begin{array}{l}\text { Determinar la prevalencia } \\
\text { de ansiedad en el grupo } \\
\text { de estudio. }\end{array}$ & $\begin{array}{l}\text { Análisis univariado: se emplearán medidas de } \\
\text { frecuencias absolutas (\#) y relativas (\%) }\end{array}$ \\
\hline
\end{tabular}




\begin{tabular}{|c|c|}
\hline $\begin{array}{l}\text { Explorar la asociación } \\
\text { entre la prevalencia de } \\
\text { ansiedad y las } \\
\text { características } \\
\text { demográficas y } \\
\text { socioeconómicas del } \\
\text { grupo de estudio. }\end{array}$ & $\begin{array}{l}\text { Análisis bivariado: se usarán pruebas paramétricas } \\
\text { y/o no paramétricas, según el cumplimiento del } \\
\text { supuesto de normalidad. Como el evento es una } \\
\text { variable dicotómica sería más preciso decir: la } \\
\text { comparación de la ansiedad con variables } \\
\text { independientes cualitativas se hará con la prueba chi } \\
\text { cuadrado de Pearson, mientras que la comparación } \\
\text { con las variables cuantitativas se realizará con las } \\
\text { pruebas t student (si se cumple el supuesto de } \\
\text { normalidad) o U de Mann Whitney (si no se cumple } \\
\text { el supuesto de normalidad). }\end{array}$ \\
\hline $\begin{array}{l}\text { Identificar las } \\
\text { características } \\
\text { demográficas y } \\
\text { socioeconómicas que } \\
\text { podrían explicar la } \\
\text { prevalencia de ansiedad. }\end{array}$ & $\begin{array}{l}\text { Análisis multivariado: se realizará regresión logística } \\
\text { binara multivariable. }\end{array}$ \\
\hline \multicolumn{2}{|l|}{ Ejemplo 2.} \\
\hline \multicolumn{2}{|c|}{$\begin{array}{l}\text { Objetivo general: Describir el perfil de calidad de vida de personas con artritis } \\
\text { atendidas en una IPS especializada de Medellín durante el } 2015 \text {, y su relación con } \\
\text { características sociodemográficas y clínicas. }\end{array}$} \\
\hline Objetivos específicos & Plan de análisis \\
\hline $\begin{array}{l}\text { Describir las } \\
\text { características } \\
\text { sociodemográficas y } \\
\text { clínicas. }\end{array}$ & $\begin{array}{l}\text { Análisis univariado: Frecuencias y medidas de } \\
\text { resumen. }\end{array}$ \\
\hline $\begin{array}{l}\text { Describir el perfil de } \\
\text { calidad. }\end{array}$ & Análisis univariado: medidas de resumen. \\
\hline $\begin{array}{l}\text { Explorar la relación entre } \\
\text { la Calidad de vida y las } \\
\text { características } \\
\text { sociodemográficas y } \\
\text { clínicas del grupo de } \\
\text { estudio. }\end{array}$ & $\begin{array}{l}\text { Análisis bivariado: pruebas paramétricas y no } \\
\text { paramétricas. } \\
\text { Como la calidad de vida es una variable cuantitativa } \\
\text { se podría poner: para comparar la calidad de vida } \\
\text { con variables dicotómicas se usarán las pruebas t } \\
\text { student o U de Mann Whitney, con las politómicas } \\
\text { Anova o H de Kruskal Wallis, con las cuantitativas } \\
\text { correlaciones de Pearson o Spearman. }\end{array}$ \\
\hline $\begin{array}{l}\text { Determinar las } \\
\text { características } \\
\text { sociodemográficas y } \\
\text { clínicas que podrían } \\
\text { explicar la calidad de } \\
\text { vida. }\end{array}$ & $\begin{array}{l}\text { Análisis multivariado: se realizará regresión lineal } \\
\text { multivariable. }\end{array}$ \\
\hline
\end{tabular}

En la metodología se deben precisar las variables con las cuales se medirán los aspectos demográficos, socioeconómicos o clínicos.

Fase metodológica: tamaño de muestra, recolección de la información, control de sesgos, cuantificación de la confusión, análisis estadístico.

Con relación a la muestra y el muestreo, se debe aclarar que el primer término alude el número de unidades de análisis que debe incluir el estudio para representar una población dada, mientras que el muestreo obedece a la forma de seleccionarlas (3).

Los estudios descriptivos, al igual que todo tipo de estudio de investigación, debe hacer la selección de una muestra de la población, por lo que resulta determinante establecer qué tamaño debe tener. Para ello se establece un grado de confianza (generalmente del 95\%), el error de muestreo (que debe estar entre 1\% y 5\%), la población de referencia y el estimador que se utilizaría, éste último corresponde a la frecuencia esperada del evento cuando la variable de respuesta es cualitativa, o la desviación estándar cuando se trata de una variable dependiente cuantitativa; estos datos deben ser extraídos de la literatura y si no es posible de la experiencia de los investigadores. Particularmente en el cálculo de tamaños de muestra en estudios cuya variable dependiente es cualitativa, si se desconoce su valor se toma un valor de 50\%, el cual proporciona el mayor tamaño de muestra posible. Finalmente, en la literatura existen múltiples programas para su cálculo, Epidat incorpora un compendio exhaustivo de técnicas comúnmente usadas en investigación epidemiológica para tal fin (7).

Para una adecuado cálculo del tamaño de muestra y del muestreo, se deben tener claramente identificados la población de estudio, las unidades de observación, muestreo y análisis, y los criterios de inclusión y exclusión, estos ultimos obedecen a características adicionales que se adscriben a la población de estudio con el fin de facilitar su selección y evitar sesgos de selección.

En la recolección de la información se debe precisar tres aspectos centrales:

1. La fuente de información, es decir, si será primaria (el investigador hace la recolección de sus variables dependientes e independientes) o secundaria (el investigador hace los análisis con base en información recolectada de forma previa al inicio del proyecto de investigación).

2. Descripción del instrumento de medición: en este se deben indicar las características básicas con las cuales se garantice la validez y reproducibilidad de la información, en este sentido, para mediciones biológicas es recomendable hace explícitas algunas características como sensibilidad y especificidad, controles de calidad, certificados de calibración o verificación; en el caso de encuestas debe indicarse su proceso de validación a la luz de características como la apariencia, el contenido, el constructo o el criterio (8).

3. Forma de obtención de mediciones, con su respectivo control de sesgos.

\section{Control de sesgos}

En esta parte se deben hacer explícitas las estrategias para garantizar la validez interna de los resultados, entendida como la minimización de los errores sistemáticos (variaciones predecibles, no aleatorias, que tienden a subestimar o sobrevalorar la medición) que se pueden 
cometer durante la selección de los sujetos de estudio o las mediciones realizadas, garantizando que los resultados son veraces y correctos para el grupo de estudio. Cabe aclarar que la validez externa depende de la interna, por lo que algunos estudios prefieren sacrificar la primera, ésta alude la posibilidad de generalizar los resultados a la población de referencia y a otras similares, lo que depende de la representatividad de la muestra estudiada (si su selección fue probabilística) $(3,9)$.

Dichos errores sistemáticos son denominados sesgos, los cuales pueden ser de selección o información:

1. Sesgos de selección: errores en la selección de las unidades de análisis; en los estudios descriptivos se pueden controlar con los criterios de exclusión, por ejemplo excluir pacientes que no cumplan las condiciones pre-analíticas para una examen de laboratorio, personas con alteraciones cognitivas o efectos de alucinógenos evidentes que impidan contestar una encuesta, personas que no firmen el consentimiento informado, o quienes exijan remuneración para participar en un estudio (9).

2. De información: aquellos que se presentan durante la medición de las variables, clasificados en los siguientes:

a. Del observador o investigador: se controla con la estandarización, la capacitación sobre todo el proyecto de investigación, o demostrando la reproducibilidad intra e interobservador de sus mediciones. El personal de investigación debe conocer a fondo los instrumentos de medición, los procedimientos a seguir cuando no se encuentre uno de los sujetos de estudio y estar estandarizado para su recolección (9). Para evitar errores en el diligenciamiento de la información se recomienda doble digitación, en este proceso se acostumbran dos tipos de verificación, por rangos (no hallar valores fuera del límite de la variable o no compatibles con la variable) o contingenciallógica (valores incoherentes, por ejemplo registro de embarazos en un hombre). Esta verificación puede hacerse en bases de datos relacionales (DBase, Paradox, Fox-Pro) o programas estadísticos diseñados para captura (recolección) de datos (Epilnfo, CDC, Atlanta) (3).

b. Del sistema de medición y los instrumentos: se controla empleando mediciones válidas, estandarizadas y con control de calidad, entre otros (9). c. De los sujetos de estudio: incluyen el sesgo de memoria, entrenamiento, fatiga (9).

Otros autores clasifican los sesgos de información en tres tipos (10):

1. En la identificación de la exposición: sesgo de memoria o del entrevistador.

2. En la identificación del desenlace: sesgo del observador o del que responde.

3. Errores de clasificación.

Además presentan sesgos combinados de información/ selección como el sesgo de vigilancia médica o de detección, sesgo temporal y otros (10).

Para determinar la confiabilidad y validez de las mediciones (controlar sesgos), tradicionalmente se ha recomendado la realización de una prueba piloto (3).

Algunos autores emplean un tercer grupo de sesgos mal llamados de confusión, lo cual es un error, debido a que la confusión no es un error sistemático. La confusión es diferente del sesgo, en el sentido que, aunque la relación no es causal si es real (indirecta, no sesgada) (10) además, las variables de confusión, como error aleatorio, varían de manera no predecible (9).

Confusión: refiere el hecho de hallar una asociación no causal entre la exposición o variable independiente y el evento de estudio, debido a la influencia de una tercera variable (o grupo de variables) denominada confusor (otra variable independiente). Para que una tercera variable independiente sea confusora debe cumplir tres requisitos: i) estar asociada con la variable independiente, ii) estar asociada con el desenlace $y$ iii) no ser una variable intermedia en la cadena causal entre la exposición y el desenlace. La tercera variable puede inducir, fortalecer, debilitar o eliminar la asociación entre una variable de exposición y el desenlace (10).

El control de la confusión puede realizarse desde el diseño, principalmente en estudios analíticos que conforman grupo equiparados por potenciales confusores. Generalmente en los estudios descriptivos se hace en el análisis de la información, dado que la selección de los sujetos de estudio se hace de la población general, para ello se hace análisis estratificado o análisis multivariante (3).

Cabe aclarar que el análisis estratificado presenta un uso restringido en la actualidad dado que sólo permite hacer el ajuste por una variable, además no es útil cuando en el análisis se representa una variable cuantitativa; a diferencia de los modelos multivariados que permiten el ajuste por un 
mayor número de variables, independientemente de su naturaleza. Adicional a ello, los modelos de regresión "permiten estimar el grado de asociación entre dicha posible causa y la respuesta ajustando por el posible efecto confusor de todas las covariables incorporadas al modelo" (10,11).

\section{Análisis de la información:}

Antes de describir las herramientas estadísticas empleadas en los análisis es oportuno presentar la clasificación de las variables.

Tabla 4. Clasificación de las variables del estudio.

\begin{tabular}{|c|c|c|}
\hline $\begin{array}{l}\text { Según la } \\
\text { naturaleza }\end{array}$ & Según el tipo & $\begin{array}{l}\text { Según nivel de } \\
\text { medición }\end{array}$ \\
\hline \multirow{2}{*}{ Cualitativas } & Dicotómica o bicategórica & Nominal \\
\hline & Politómica & Ordinal \\
\hline \multirow{2}{*}{ Cuantitativas } & Discreta & Intervalo \\
\hline & Continua & Razón \\
\hline
\end{tabular}

El análisis univariado se realizan frecuencias absolutas (recuento) o relativas (proporción o porcentajes) para las variables cualitativas, mientras que para las cuantitativas se calculan medidas de resumen en sus cuatros grupos: i) tendencia central (media, mediana y moda), ii) dispersión o variabilidad (varianza, desviación estándar, rango, rango intercuartil, coeficiente de variación), iii) posición (percentiles, deciles, cuartiles) y iv) forma o distribución (curtosis o asimetría) $(12,13)$.

Además, si la variable desenlace es una enfermedad, su frecuencia se puede medir con incidencia o prevalencia, según el tipo de estudio (transversal o longitudinal) y de enfermedad (aguda o crónica), para este caso se recomienda consultar textos básicos de epidemiología sobre la medición de la ocurrencia de la enfermedad (14).

Con base en esta definición un estudio sobre la frecuencia de una enfermedad puede realizarse con casos incidentes o prevalentes por lo que la frecuencia sería un concepto más amplio que el de prevalencia, al incluir tantos los estudios de prevalencia como los de incidencia (10). Sin embargo, algunos autores han separado los estudios de frecuencia y de prevalencia como si fuesen dos asuntos diferentes, argumentando que la prevalencia solo puede establecerse en estudio de base poblacional o con muestreos probabilísticos, en caso de estudios con muestreos por conveniencia se debería hablar sólo de frecuencia, es decir, en esta segunda postura la prevalencia sería un concepto de mayor complejidad que el de frecuencia.

A pesar de lo anterior, no existen referencias concluyentes para aludir la frecuencia como una medida diferente a prevalencia, en una búsqueda en PubMed con la palabra Prevalence (restringida al título) se obtuvieron 48.681 resultados, revisando los primeros 100 se hallan estudios nominados como prevalencia de punto en muestras no probabilísticas, mientras que con la palabra frequency en título se obtienen estudios realizados en la población de referencia (no en muestra); lo que pone de manifiesto que la diferencia entre prevalencia y frecuencia no está unificada entre los investigadores.

Análisis bivariado: se realiza la prueba de normalidad para cada par de variables en las que se desee evaluar asociación, entre el listado de pruebas de normalidad existente las más empleadas son la prueba de Kolmogorov-Smirnov con corrección de la significación de Lillierfors, considerada una de las más potentes para muestras mayores de 50 casos. La prueba de Shapiro-Wilk está considerada como la prueba más potente para muestras entre 3 y 50 sujetos, aunque en algunas ocasiones como en datos discretos puede extenderse su uso hasta 5.000 datos (15).

Interpretación: cuando se hace la prueba de normalidad el software genera un valor $\mathrm{p}$, este se contrasta con el error alfa (complemento del grado de confianza) así:

1. Dado que generalmente se hacen análisis con una confianza del 95\%, o lo que es igual una significación del $5 \%(0,05)$, si el valor $\mathrm{p}$ de la prueba de normalidad es mayor o igual a 0,05 indicará que los datos provienen de una población con distribución normal; en consecuencia, se harán pruebas paramétricas para el análisis bivariado.

2. Si el valor $\mathrm{p}$ da un resultado menor a 0,05 indicará que los datos no provienen de una población con distribución normal; en consecuencia, se harán pruebas no paramétricas para el análisis bivariado.

Luego de realizar la prueba de normalidad, se toma la decisión sobre la prueba paramétrica o no paramétrica que serviría para explorar la asociación, así $(3,16)$.

Tabla 5. Plan de análisis bivariado: pruebas paramétricas y no paramétricas para muestras independientes y pareadas.

\begin{tabular}{|l|l|l|l|}
\multicolumn{1}{c}{$\begin{array}{l}\text { Pruebas } \\
\text { paramétricas }\end{array}$} & \multicolumn{2}{c|}{$\begin{array}{l}\text { Pruebas no } \\
\text { paramétricas }\end{array}$} \\
$\begin{array}{l}\text { Naturaleza o } \\
\text { Tipo de variables } \\
\text { que se comparan }\end{array}$ & $\begin{array}{l}\text { Muestras } \\
\text { independientes }\end{array}$ & $\begin{array}{l}\text { Muestras } \\
\text { independientes }\end{array}$ & $\begin{array}{l}\text { Muestras } \\
\text { pareadas }\end{array}$ \\
\hline Dos cualitativas & Prueba Z & $\begin{array}{l}\text { Chi cuadrado o } \\
\text { Fisher }\end{array}$ & $\begin{array}{l}\text { C Nemar, } \\
\text { Q de Cochran }\end{array}$ \\
\hline Dos cuantitativas & $\begin{array}{l}\text { Coeficiente de } \\
\text { correlación de } \\
\text { Pearson }\end{array}$ & $\begin{array}{l}\text { Coeficiente de } \\
\text { correlación Rho } \\
\text { de Spearman }\end{array}$ & Tau de Kendall \\
\hline $\begin{array}{l}\text { Cuantitativa con } \\
\text { dicotómica }\end{array}$ & $\begin{array}{l}\text { t Student para } \\
\text { muestras } \\
\text { independientes }\end{array}$ & $\begin{array}{l}\text { Prueba U de } \\
\text { Mann Whitney }\end{array}$ & Wilcoxon \\
\hline $\begin{array}{l}\text { Cuantitativa con } \\
\text { politómica }\end{array}$ & $\begin{array}{l}\text { Anova de una vía } \\
\text { (o de un factor) }\end{array}$ & $\begin{array}{l}\text { Prueba H de } \\
\text { Kruskal Wallis }\end{array}$ & Friedman \\
\hline
\end{tabular}


Fisher: aplica cuando las dos variables son dicotómicas y en la tabla de contingencia $(2 \times 2)$ se presenta una frecuencia esperada menor que cinco.

C Nemar: aplica cuando las dos variables son dicotómicas.

Q de Cochran: aplica cuando en las dos variables se presenta al menos una politómica.

Muestras pareadas: cada individuo es su propio control, es decir, para estudios prospectivos en los que se compara la medición de una persona frente a la medición de la misma variable en el mismo individuo en un momento anterior. Aunque las pruebas para muestras pareadas son poco usadas en estudios descriptivos, se agregaron en esta tabla para que el elector tenga una visión amplia de las opciones para hacer análisis bivariado.

Si en la comparación de dos variables cualitativas, una de ellas es ordinal se aplica la prueba chi cuadrado de tendencial lineal.

Interpretación: si en cualquiera de las pruebas anteriores se observa un valor $\mathrm{p}$ menor a 0,05 , será indicador de asociación estadística (de carácter exploratorio para los estudios descriptivos) entre las dos variables analizadas.

Se debe aclarar que una de las pruebas aplicada en la estadística paramétrica, ANOVA, además de tener el supuesto de normalidad, tiene el de homocedasticidad $\mathrm{u}$ homogeneidad de varianzas, que se evalúa con el estadístico de Levene.

En un texto posterior, se describirá en detalle cada una de estas pruebas, y otras como las pruebas posthoc y correlaciones de variables cualitativas, en conjunto con su ejecución en SPSS.

El análisis bivariado podría complementarse con algunas medidas epidemiológicas útiles para comparar dos variables dicotómicas, es decir, cuando la exposición y el desenlace se miden como presencia-ausencia, estas medidas son la razón de prevalencias (RP) y las razones de odds (OR), las cuales se interpretan de la forma siguiente:

1. Si la RP o la OR da 1,0 o su intervalo de confianza incluye el 1 , indica que no existe asociación entre las dos variables.

2. Si es menor a 1 (y su intervalo no incluye este valor) indica que la presencia del factor de exposición disminuye la ocurrencia de la enfermedad, es lo que se conoce como factor protector.

3. Si es mayor a 1 (y su intervalo no incluye este valor) indica que la presencia del factor de exposición incrementa la ocurrencia de la enfermedad, es lo que se conoce como factor de riesgo.

Adicional a lo anterior, se pueden consultar algunos artículos que de manera sucinta, han descrito las medidas tradicionales para hacer análisis univariado y bivariado, en los cuales se presentan conceptos como variable y sus niveles de medición; diferencias entre proporciones, razones y tasas; medidas de frecuencia como la prevalencia o la incidencia; medidas de asociación (razones de prevalencia, de odds y riesgo relativo), y medidas de impacto (riesgo atribuible, fracción prevenible) (12-13).

Análisis multivariado: se pueden hacer tres tipos de regresiones en los estudios descriptivos, dependiendo de la naturaleza y el tipo de variable que sea el evento de estudio:

1. Logística binaria: cuando la variable de respuesta es dicotómica.

2. Logística multinomial: cuando la variable de respuesta es politómica.

3. Lineal: cuando la variable de respuesta es cuantitativa.

Cabe aclarar que las regresiones pueden realizarse con fines predictivos, explicativos o de ajuste, su uso más común en estudios descriptivos es el último, en este caso los pasos para ejecutarlo son:

1. Seleccionar las variables independientes: si sólo se desea determinar si las asociaciones detectadas en el análisis bivariado son reales o están "confundidas", las variables independientes serían aquellas que en el análisis bivariado presenten valor p menor a 0,05, se mira el modelo multivariable cuales conservan la significación luego del ajuste por las demás variables relacionadas (indicador de asociación real) y cuales no (indicarían que la asociación bivariada era producto de una variable confusora) (11).

$\mathrm{Si}$ se desea identificar las variables que podrían explicar la ocurrencia del evento, se tomarían como variables independientes todas las que teóricamente sean relevantes y aquellas que en el análisis bivariado hayan presentado valores menores a 0,25 .

2. Validar los supuestos del modelo.

Supuestos de la regresión lineal: aleatoriedad de Y con la prueba de las rachas (su valor $\mathrm{p}$ debe ser mayor a ,005) o garantizándola desde a recolección de la información; linealidad con ANOVA (el valor $\mathrm{p}$ 
debe ser menor a 0,05), la normalidad de los residuos, la varianza constante de los residuos (con el método gráfico), la no correlación de los residuos con la prueba de Durbin Watson, la no colinealidad o multicolinealidad con la estimación de la tolerancia de cada variable y el factor de inflación de la varianza FIV y la significación estadística de los coeficientes de regresión.

Regresión logística: se evalúa la bondad de ajuste con el estadístico de Hosmer-Lemeshow y sus supuestos básicos son: valor esperado del error debe ser cero, que no haya autocorrelación, que no haya correlación entre el error y las variables independientes y que no haya multicolinealidad entre las variables independientes (18).

3. Interpretar los coeficientes de regresión como el cambio que se genera en la variable dependiente por cada unidad de cambio de la independiente, ajustando por las demás variables del modelo $(10,17)$.

\section{Fase de divulgación: contenido del artículo y guía STROBE.}

En el reporte de los estudios descriptivos se recomienda la aplicación de la guía STROBE (Strengthening the Reporting of Observational studies in Epidemiology), la cual contiene 22 puntos distribuidos así: uno para título y resumen, dos en introducción, nueve en los métodos, cinco en resultados, cuatro en discusión y uno sobre las fuentes de financiación del estudio (18).

En general, algunos aplican estos puntos como una lista de chequeo para los artículos escritos, pero la idea es que previo a la escritura del informe final se revisen estos criterios para hacer más eficiente esta etapa final de una investigación.

Adicional a esto se presentan algunos elementos comunes a la escritura de los artículos derivados de estudios descriptivos en la siguiente tabla.

Tabla 6. Componentes de una artículo de investigación descriptivo

\begin{tabular}{|l|c|}
\hline Título & $\begin{array}{l}\text { Tema con su delimitación poblacional, espacial y temporal, } \\
\text { antecedido por el término Prevalencia, Incidencia o Frecuencia. }\end{array}$ \\
\hline Autores & Según indicaciones de la revista en la cual se desea publicar. \\
\hline Resumen & $\begin{array}{l}\text { Generalmente es estructurado, es decir, desagregado en } \\
\text { introducción, objetivo general, métodos, resultados y conclusión. }\end{array}$ \\
\hline 1. & $\begin{array}{l}\text { Definición del evento o tema de estudio. } \\
\text { Presentación de la importancia de estudiarlo, puede ser por } \\
\text { sus implicaciones clínicas (deterioro de la salud física o } \\
\text { psicológica, complicaciones, daño en la funcionalidad, } \\
\text { incapacidades), epidemiológicas (alta ocurrencia, muchas } \\
\text { personas en riesgo, alta letalidad o mortalidad, múltiples } \\
\text { factores de riesgo) o económicas (costos de su atención, } \\
\text { intervención o prevención). }\end{array}$ \\
\hline
\end{tabular}

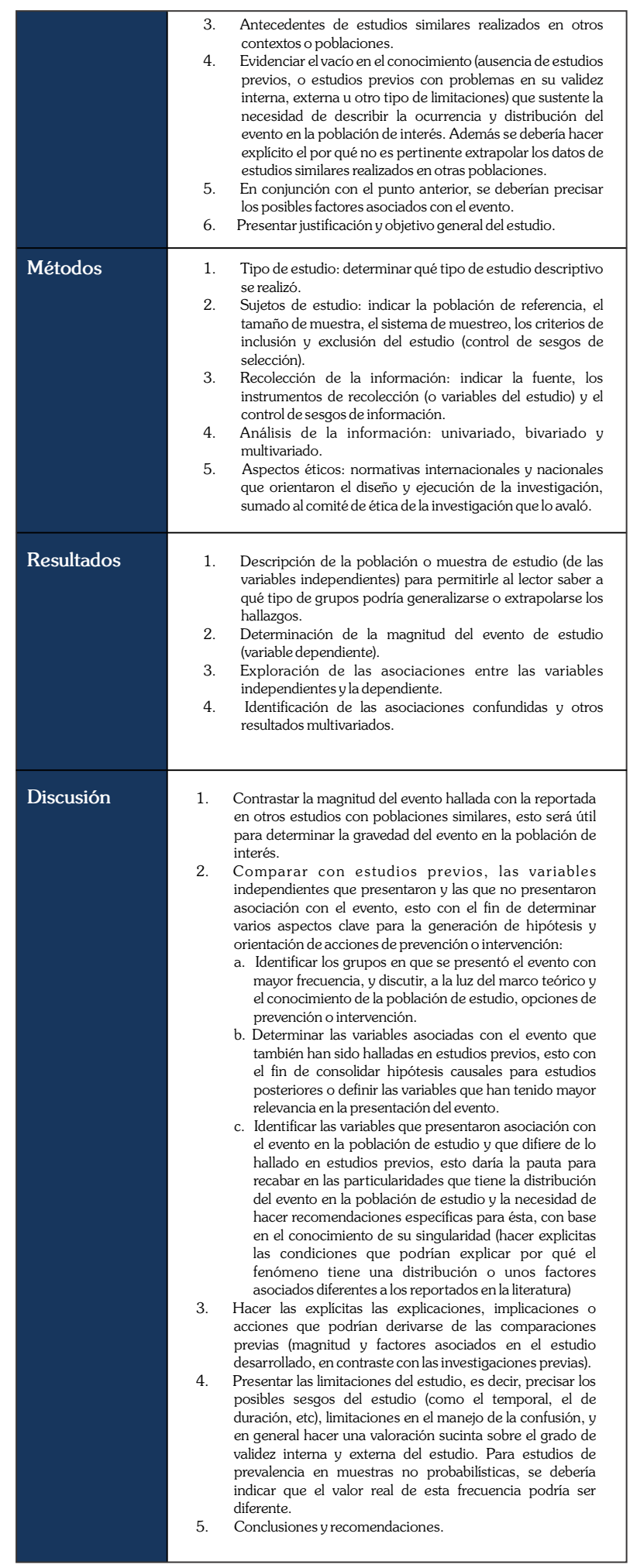


Para evaluar los elementos comunes de los estudios descriptivos y algunas particularidades que dependen del tema de estudio, se sugiere la lectura de algunos artículos que el autor ha realizado en esta modalidad de investigación y que cristalizan el contenido expuesto previamente (1922).

\section{Discusión}

Este artículo expuso las consideraciones conceptuales y metodológicas centrales de los estudios descriptivos, para que los investigadores interesados puedan disponer de una recopilación de las exigencias teóricas, epidemiológicas, estadísticas, de metodología de la investigación y de divulgación del conocimiento científico; con el ánimo de mitigar algunos calificativos peyorativos que ha recibido esta modalidad de investigación, y la pretensión de recabar sobre su necesidad y pertinencia.

Se muestra que la complejidad de los estudios descriptivos los aproxima a los analíticos, lo que vindica la crítica a los programas académicos, revistas y demás comunidades que los rechazan a ultranza. También sirve como base para evitar un uso excesivo de la nominación de estudios analíticos en investigaciones cuya estructura básicamente es descriptiva pero por hacer análisis con pruebas de hipótesis y regresiones o por comparar varios grupos, los autores o editores les incrementan su verdadero nivel de complejidad.

En resumen, la investigación descriptiva usa los datos para determinar cómo la ocurrencia o presentación del evento varía en función de las características de base de la población de estudio; cuando la ocurrencia no es uniforme se identifican posibles grupos de riesgo con propósitos preventivos; se generar hipótesis causales bajo el "paradigma clásico agente-huesped-medio" (10) y se proponen acciones de atención o prevención que sean coherentes con las características de base de las poblaciones de interés (evitar el uso descontextualizado de evidencia generada en otros contextos y aplicada de forma acrítica a todo tipo de poblaciones).

Como conclusión, el artículo presentó la fundamentación conceptual de los estudios descriptivos y algunas consideraciones operativas que tradicionalmente resultan de difícil comprensión al estar atomizadas en textos de epidemiología, estadística o metodología de la investigación, o porque los "expertos" son muy puristasrígidos-acríticos en la aplicación de sus principios metodológicos o estadísticos. La investigación descriptiva es la que expone de manera más veraz y directa la realidad de un fenómeno de estudio y su distribución en lugares, momentos y comunidades específicas; por ello, es necesario conocer sus exigencias conceptuales y operativas para mitigar concepciones erradas sobre su alcance.

\section{Conflicto de intereses}

Los autores declaran no tener de manera directa o indirecta, ningún tipo de conflicto de intereses financieros, académicos o laborales que puedan poner en peligro la validez de este estudio.

\section{Financiación}

Este trabajo se realizó con el apoyo financiero de la Universidad de Antioquia y la Universidad Cooperativa de Colombia (Estrategia Sostenibilidad 2014).

\section{Referencias}

1. J. Cardona Reconocimiento de la utilidad de la epidemiología descriptiva. Medicina \& Laboratorio. 2013;19(3-4):109-10.

2. Rada G, Merino T, Universidad Católica de Chile. Estudios descriptivos: Tipología. 2007. [Sitio en internet]. Disponible en: http://escuela.med. puc.cl/recursos/recepidem/epiDesc4. Acceso en abril de 2014.

3. Hernández B, Velasco H. Encuestas transversales. Salud pública de México. 2000;42(5):447-55.

4. Rada G, Merino T, Universidad Católica de Chile. Estudios de incidencia. 2007. [Sitio en internet]. Disponible en: http://escuela.med.puc.cl/ recursos/recepidem/ epiDesc2.htm. Acceso en abril de 2014.

5. Rada G, Merino T, Universidad Católica de Chile. Estudios ecológicos. 2007. [Sitio en internet]. Disponible en: http://escuela.med.puc.cl/ recursos/recepidem/ epiDesc5.htm. Acceso en abril de 2014.

6. Hernández R, Fernández C, Baptista P. Metodología de la investigación. Cuarta Edición. México: McGraw-Hill. 2006.

7. Xunta de Galicia, Organización Panamericana de la Salud. Epidat Programa para Análisis Epidemiológico de Datos Tabulados. Ayuda Muestreo.

8. Ramada J, Serra C, Delclós G. Adaptación cultural y validación de cuestionarios de salud: revisión y recomendaciones metodológicas. Salud pública de México. 2013;55(1):57-66.

9. Hernández M, Garrido F, Salazar E. Sesgos en estudios epidemiológicos. Salud pública de México. 2000;42(5):438-46.

10. Szklo M, Nieto J. Epidemiologia intermedia: conceptos y aplicaciones. España: Ediciones Díaz de Santos. 2003; p. 435.

11. Silva L. Una Ceremonia Estadística para Identificar Factores de Riesgo. Salud Colectiva, Buenos Aires. 2005;(3):309-22.

12. Moreno A, López S, Corcho A. Principales medidas en epidemiología. Salud pública de México. 2000;4(4):337-48.

13. Fernández P. Uso de la estadística y la epidemiología en atención primaria. En: Gil VF, Merino J, Orozco D, Quirce F. Manual de metodología de trabajo en atención primaria. Universidad de 
Alicante. Madrid, Jarpyo Editores, S.A. 1997;115-161.

14. Gordis L. Epidemiology. 3. ed. Estados Unidos: Elsevier Science. 2004. p.335

15. IBM SPSS Statitisc. Ayuda, Manual del usuario SPSS. Pruebas de normalidad. [Sitio en internet]. Disponible en: http://emic130083:50279/ help/index.jsp?topic=/ com.ibm.spss.statistics.help/overvw_auto_0.htm. Acceso en abril de 2014

16. Gómez M, Danglot C, Vega L. Sinopsis de pruebas estadísticas no paramétricas. Cuándo usarlas. Revista Mexicana de Pediatría. 2003;70(2):91-9.

17. SPSS Líderes en análisis predictivo. Análisis estadístico avanzado con IBM ${ }^{\circledR}$ SPSS Statistics. Regresión logística binaria y multinomial. SPSS Andino: Infórmese Ltda. p. 9-93.

18. Von E, Altmanb D, Eggera M, Pocockd S, Gotzschee P, Vandenbrouck J. Declaración de la Iniciativa STROBE (Strengthening the Reporting of Observational studies in Epidemiology): directrices para la comunicación de estudios observacionales. Gac Sanit. 2008;22(2):144-50.

19. Patiño J, Cortés M, Cardona J. Seroprevalencia de marcadores de infecciones transmisibles por vía transfusional en banco de sangre de Colombia. Revista de Salud Pública. 2012;46(6):9509.

20. Cardona J, Peláez L, López J, Duque M, Leal O. Calidad de vida relacionada con la salud en adultos con $\mathrm{VIH} /$ sida de Medellín, Colombia. Biomédica: Revista del Instituto Nacional de Salud. 2011;31(4): 532-44.

21. Cardona J, León V, Cardona A. Estado de salud y calidad de vida en pacientes con fibromialgia, Medellín. Revista Colombiana de Reumatología. 2014; 21(1):10-20.

22. Cardona J, Llanes O. Hipertensión arterial y sus factores en indígenas Emberá-Chamí. CES Medicina. 2013;27(1):31-43. 